Archives

$17 \mid 1996$

Hommage à Bernard Lepetit

\title{
Un modèle pour l'architecture américaine de 1893 à 1939. Les thermes romains
}

\section{Pascal Laurent}

\section{(2) OpenEdition}

Journals

\section{Édition électronique}

URL : http://journals.openedition.org/ccrh/2628

DOI : $10.4000 /$ ccrh. 2628

ISSN : $1760-7906$

Éditeur

Centre de recherches historiques - EHESS

Édition imprimée

Date de publication : 4 octobre 1996

ISSN : 0990-9141

Référence électronique

Pascal Laurent, «Un modèle pour l'architecture américaine de 1893 à 1939. Les thermes romains », Les Cahiers du Centre de Recherches Historiques [En ligne], 17| 1996, mis en ligne le 27 février 2009,

consulté le 10 décembre 2020. URL : http://journals.openedition.org/ccrh/2628 ; DOI : https://doi.org/ $10.4000 /$ ccrh. 2628

Ce document a été généré automatiquement le 10 décembre 2020.

Article L.111-1 du Code de la propriété intellectuelle. 


\title{
Un modèle pour l'architecture américaine de 1893 à 1939. Les thermes romains
}

\author{
Pascal Laurent
}

Après une expérience professionnelle de plusieurs années dans le domaine de l'architecture, j'ai songé à entreprendre une thèse à l'École des hautes études en sciences sociales. J'ai rencontré Bernard Lepetit en novembre 1993. Il m'a encouragé à entreprendre un DEA, sous sa direction. Alors que je partais d'une idée très générale sur l'architecture des Beaux-Arts, son aide, concise, claire et efficace, m'a peu à peu amené à préciser un sujet de thèse :» L'influence des thermes romains sur l'architecture américaine de 1893 à 1939 ». Le texte qui suit est extrait de la plaquette que j'ai donnée, le 15 mars 1996, pour la section architecture du concours de la villa Médicis. Je n'ai pas été sélectionné, mais ce document a l'avantage de reprendre les grandes lignes de ma thèse tout en résumant l'état de mes recherches à la veille du décès de Bernard Lepetit. C'est en effet le dernier texte que je lui ai présenté et il s'est montré très heureux de ma démarche : puisque j'étudiais les prix de Rome en architecture, il lui est apparu logique que j'essaie de m'identifier à mon sujet.

\section{Dioclétiomanie}

1 En 1900, le critique français E. Rivolan ${ }^{1}$ décrit les concours de l'École des beaux-arts comme atteints de»dioclétiomanie» et de»caracallomanie». Alors qu'en France ce phénomène reste limité aux projets d'école, cette» thermo-manie » atteint les États-Unis, où les architectes les plus reconnus sont aussi les plus romanophiles. Nombre de gares de chemins de fer ne sont-elles pas des copies avouées de thermes? C'est aussi le cas, mais d'une façon plus discrète, d'Ellis Island, de la Galerie nationale, de nombreux musées, de banques, de gymnases, de campus et de pavillons d'expositions universelles. Ainsi la visite 
de l'ancienne bibliothèque de Chicago fait que» l'on s'attend plutôt à trouver, dans ce décor, des étuves et des douches $»^{2}$... que des livres.

Cette» thermo-manie » culmine en France dans les années 1840-1850. Les projets des grands prix sont prétextes à l'utilisation d'un style romain directement influencé par les découvertes archéologiques rendues publiques par les envois des étudiants pensionnaires de la villa Médicis à Rome. Mais l'on construit alors trop peu de monuments pour que le décalage entre l'enseignement et la réalité soit évident. Ce sont les travaux d'Haussmann qui vont révéler une réalité, un contexte nouveau tant dans les programmes que dans l'environnement. D'un style néo-classique nous allons passer à un style Beaux-Arts qui s'assouplira, jusqu'à l'apothéose de l'Art nouveau à l'Exposition de 1900. Pourtant, les thermes romains restent le thème majeur de l'enseignement de l'École des beaux-arts et l'exercice favori des concours et des prix d'émulation. Julien Guadet les considère comme l'équivalent romain de la perfection classique grecque représentée par le Parthénon. Le thème de l'établissement thermal est donné 47 fois en sujet de concours entre 1819 et 1914. Pourtant, en dehors des thermes du Mont-Dore, de Royat et de Châtel-Guyon, aucun bâtiment construit vers 1900 ne peut être comparé aux thermes romains. Seul le Grand Palais à Paris sera parfois surnommé» Terminus Caracalla", mais il s'agit là d'un bâtiment directement influencé par l'exposition de Chicago de 1893.

3 Le langage des thermes est celui d'une démocratie prétendant à un impérialisme démagogique. Il est l'instrument le plus efficace d'une politique sociale, la traduction d'un évergétisme, que ce soit de la part d'un empereur romain ou de celle des grandes compagnies ferroviaires ou bancaires américaines. Ce langage vient recouvrir de nouveaux programmes qui n'ont pas de précédents architecturaux. Ainsi certains critiques trouveront même que les voûtes d'arêtes de la salle des pas perdus de Pennsylvania Station à New York (copie des thermes de Caracalla en $25 \%$ plus grand) sont davantage appropriées à une gare $\mathrm{du} \mathrm{xx}^{\mathrm{e}}$ siècle qu'à des thermes du $\mathrm{II}^{\mathrm{e}}$ siècle. C'est dans cet esprit qu'après 1910 certains projets d'école, aux États-Unis, proposent comme programme une salle de cinéma, un champ d'aviation, une usine de voitures... cependant que les projets continuent de se référer à un répertoire de formes antiques très souvent thermales.

4 Aussi pouvons-nous parler des lobbies des premiers gratte-ciel. Ils peuvent être associés au frigidarium des thermes romains, qui n'avait pas de fonction propre si ce n'est celle d'être un lieu de passage, une place intérieure (il n'est pas décoré d'un escalier, comme c'est le cas des grands halls en France), et dont le propos était d'être un refuge à l'écart du désordre urbain. Ainsi, le propre des Américains n'est pas d'avoir copié les voûtes d'arêtes du frigidarium, mais d'avoir vu la nécessité d'un lieu sans fonction prédéterminée.

En plus du décorum, on retrouve dans les premiers halls américains une ampleur antique qui m'a fait remarquer que, dans leur espace interne, les Romains, contrairement aux chrétiens de l'époque gothique, ne cherchaient pas la hauteur mais l'ampleur. À la dominante verticale, ils préféraient exagérer l'effet de portée horizontale. Je suis actuellement en train d'étudier l'évolution presque organique du lobby américain, qui, partant de la forme horizontale typiquement basilicale, va devenir une grande cour verticale au centre des gratte-ciel. Autre similitude, les thermes s'organisaient comme une progression interne des espaces qui menaient au frigidarium. C'est très souvent ainsi qu'est conçu le lobby américain qui n'est généralement pas situé à l'entrée du bâtiment mais au cœur de celui-ci. Il s'agissait là d'une hiérarchie qui a peu été reprise par les intérieurs Beaux-Arts français. Ainsi, le propre de certains monuments serait de dépasser 
l'intention des architectes pour contenir, dès leurs origines, une multipotentialité sousjacente. En d'autres termes, de pouvoir faire perdurer leur forme à leur fonction. En cela, les thermes sont les monuments des monuments et, en les reprenant à leur compte, les architectes américains les considéraient non pas comme exhaustifs mais comme exemplaires.

C'est aller là à l'encontre de Quatremère de Quincy pour qui les thermes étaient le plus beau rapport entre la forme et la fonction. En fait, au tournant du siècle, ils sont devenus une forme hors contexte à laquelle il faut trouver des prétextes pour la restituer. Mais, très souvent, un bâtiment placé hors de son contexte révèle des paramètres et des lois intrinsèques à sa forme qui n'apparaissent pas comme évidentes sur le lieu d'origine. Ainsi, les différences entre les thermes romains sont amplifiées lorsque ceux-ci sont réimplantés outre-Atlantique. Les oppositions entre un classicisme vitruvien et un baroque sévérien $\mathrm{du} \mathrm{III}^{\mathrm{e}}$ siècle se retrouvent dans les dichotomies du début de ce siècle. Il semblerait même que si Rivolan parle indifféremment de "dioclétiomanie » et de» caracallomanie ", ce soit là deux manies à différencier aux États-Unis où, pour chaque grande gare et pour chaque exposition universelle, on peut faire correspondre un des thermes antiques majeurs. Ceux-ci semblent être l'élément clé permettant de comprendre à la fois l'architecture romaine, l'enseignement français et une certaine société américaine.

7 Modèle organisateur de toutes les formes romaines, les thermes sont intéressants en tant que globalité romaine. L'enseignement Beaux-Arts imposait le plan, à chacun d'élever la façade de son style. Les Français n'ont pas reconstruit Rome, si les Américains l'ont fait, est-ce par choix ou par contrainte urbaine?

\section{American vitruvius}

8 L'exposition de Chicago de 1893 est l'élément révélateur du renouveau de l'architecture classique aux dépens du mouvement moderne; mais tandis qu'en 1933, pour Ernest Graham, elle inaugurait» the greatest era of building in the world's history", en 1893, Louis H. Sullivan prédisait qu'elle allait être à l'origine de» damage [which] will last for half a century from its date if no longer $\|^{3}$.

9 Ces cinquante années de» damage ", 1892-1942, sont la période qui me préoccupe. Les études la concernant ont essayé de saisir en quoi les modernes avaient échoué, mais peu ont essayé de comprendre le succès des classiques. Il existe, parmi ces derniers, une dichotomie essentielle entre une architecture Beaux-Arts romano-baroque française et une architecture Beaux-Arts romano-classique américaine. Ainsi, dans la Rome des années 1740 , les académies française et italienne vont s'opposer d'une manière assez comparable à ce qui définit la séparation entre Américains et Français en 1900. C'est-àdire que, dans les deux cas, on retrouve un rejet des tendances baroques par les premiers et par conséquent un retour au classicisme de leur part.

Par exemple, le mémorial de Lincoln, construit à Washington en 1911, est considéré par les architectes américains comme un modèle de l'architecture classique; pourtant, certains notent le fait qu'il aurait pu être construit en France à la fin de XVIII ${ }^{e}$ siècle. Il arrive d'ailleurs parfois que Piranèse soit décrit comme le Hugh Ferris du XVIII siècle et non l'inverse... En réaction, l'Art nouveau de 1900 est décrit par les critiques américains comme un» Decadent taste of fin-de-siècle France $»^{4}$. 
11 Ce tournant de siècle, l'Amérique le nomme Gilded Age ${ }^{5}$ et c'est là une description lucide d'une société qui cherche à retrouver un Golden Age ${ }^{6} \mathrm{~d}^{\prime}$ un autre temps. L'identification de la nation à l'Antiquité classique va très loin, tel l'exemple de l'Exposition de 1900, où les États-Unis sont les seuls à ne pas avoir un pavillon de style national mais une copie du Panthéon romain. Autre exemple, ce livre distribué dans les écoles de Chicago en 1912 et qui, par des illustrations des thermes de Caracalla, explique aux enfants en quoi leur ville, tout en projetant d'être un Paris sur le lac Michigan, se reconnait comme héritière de la Rome antique. Dans les années vingt, la tendance persistera avec la publication de The American Vitruvius.

Certes, comme l'Empire romain, les États-Unis ont dû intégrer de nouvelles terres, de nouveaux peuples et de nouveaux styles artistiques, mais, contrairement à Rome, les territoires conquis n'étaient pas ceux des peuples émigrants qui, eux-mêmes, n'étaient pas à l'origine de la multiplicité des styles (fait propre au XIX ${ }^{e}$ siècle). Alors, anachronisme ou paradoxe ? Toujours est-il que, pour unifier leur continent, les États-Unis ont romanisé leur territoire et que, pour construire leurs édifices, dans la seule période allant de 1900 à 1915, ils ont utilisé autant de marbre que tout l'Empire romain pendant toute sa durée.

En 1923, Mies Van der Rohe déclare que l'architecture est la volonté d'une époque transplantée dans l'espace. Au début du siècle, les architectes disposent, aux États-Unis, d'un pouvoir peu commun, mais jusqu'à quel point peuvent-ils influencer une société si celle-ci ne les soutient pas ? Que représente cette» thermo-manie » américaine ? Effet de mode? Fantasme d'architecte? Identification de toute une société à la Rome antique? Certes, les États-Unis ne construisent pas que néo-romain, mais le simple fait qu'ils le fassent, et non les autres pays, est déjà intéressant en soi. L'essentiel n'est pas de voir des similitudes entre l'Amérique et Rome mais de comprendre que certains Américains en aient vu et y aient cru. Par leurs monuments, ils ont tout fait pour laisser croire qu'ils étaient les nouveaux Romains. En fait, par une étude approfondie de l'architecture et de l'urbanisme, il me semble finalement plus intéressant de savoir en quoi ils ne l'étaient pas.

\section{Importance de la ville de Rome}

14 D'une manière générale, l'architecture qui nous concerne est Beaux-Arts, c'est-à-dire qu'elle est étudiée à Rome et enseignée à Paris. Alors, éclectisme ou historicisme? baroque ou classique? Grèce ou Rome? Tout cela s'est, en fait, constitué par des allerretour de modèles entre la France et les États-Unis avec le passage obligé, Rome.

Si les bâtiments fin de siècle français déroutent les architectes américains, ceux-ci admirent les restaurations archéologiques des pensionnaires de la villa Médicis. Il est reconnu, par exemple, que la restauration des thermes de Dioclétien par Edmond Paulin ${ }^{7}$ a grandement influencé les rendus-perspectives des plans de Grand Central Station à New York. C'est aussi sur le modèle établi par la France à la villa Médicis qu'est créée l'académie américaine à Rome à la fin du $\mathrm{XIX}^{\mathrm{e}}$ siècle. Les pensionnaires de cette nouvelle école vont restaurer à leur tour les monuments antiques romains, mais avec des consignes très strictes sur ce qui doit être considéré comme de la bonne architecture.

Beaucoup de similarités sont notables entre Rome et les villes des États-Unis. Ainsi, la gare principale de Rome est reliée par la via di Termini à la place de la République, qui constitue une entrée majestueuse dans la ville par le nord. Cette place reprend la forme et 
l'emplacement de l'exèdre des thermes de Dioclétien qui sont ainsi réunis par un axe direct (la via Nazionale) au Forum et au Capitole. Cette forme en exèdre s'ouvrant sur un axe est maintes fois réinterprétée aux États-Unis où, dans de vastes compositions urbaines, la gare prend la forme et l'emplacement des thermes de Dioclétien. Ces gares deviennent des portails monumentaux propres à l'esprit du mouvement de la» City Beautiful». C'est dans cet esprit qu'en 1906 la gare de Washington reprend ouvertement les grandes lignes des thermes de Dioclétien, à la fois dans leur état originel mais aussi dans la volumétrie de leurs ruines. Cette gare est axée sur la colonnade du dôme du Capitole fédéral américain. Retour des choses? En 1911, les Italiens construisent le monument de Victor-Emmanuel, lui aussi une vaste colonnade, établi sur le mont Capitole et dans l'axe des thermes de Dioclétien, restaurés à l'occasion de l'exposition internationale.

\section{Recherches romaines}

Personnage clé du mouvement romain, Burnham, que Charles Moore décrit comme»a Roman of the Augustinian age " ${ }^{8}$, est l'organisateur de l'exposition de Chicago de 1893. Autre figure importante, McKim est le principal fondateur de l'académie américaine à Rome. Plus que Paris, cette ville était leur référence et tandis que McKim (pour son projet de Pennsylvania Station, à New York) demandait à des figurants de simuler des mouvements de foules dans les ruines des thermes de Caracalla, Burnham étudiait les bains de Dioclétien pour esquisser la volumétrie de la gare de Washington et peut-être établir les premières esquisses du grand plan de Chicago.

Autre moment à approfondir : les expositions italiennes de 1911 qui mettent en valeur les différentes tendances architecturales de la Péninsule. Les pavillons de Turin ont une exubérance française tandis que les palais de Rome ont l'impérialisme antique qui plaît à l'Amérique. Y a-t-il eu participation des Américains à la préparation de l'exposition?

Les revues américaines de l'époque rendent compte de tout ce qui se passe à Rome concernant l'archéologie, mais il n'est presque jamais fait mention de l'Académie italienne ou de l'opinion des architectes italiens en général. Lorsqu'une école romaine est mentionnée, il s'agit presque toujours de l'Académie américaine ou de la villa Médicis. Quels rapports entretenaient toutes ces personnes de diverses nationalités lorsqu'elles étaient réunies à Rome? Quels étaient les échanges au sein des milieux intellectuels et, notamment, quelle était la notoriété de l'académie Saint-Luc au tournant du siècle ${ }^{9}$. Qui étaient ces Italiens qui ont construit la Galerie nationale, la piazza di Termini, le Palais de justice, le monument de Victor-Emmanuel, les pavillons de l'exposition de 1911...? Pourquoi la gare de Milan est-elle une» thermo-manie » à l'italienne ? Comment ont réagi les académies étrangères face à la montée du fascisme de Mussolini, dont la volonté de se référer à la Rome antique n'avait d'égale que celle des architectes américains? Par conséquent, quels furent les concepts qui contribuèrent à la création de la troisième Rome, cette utopique exposition universelle qui aurait dû avoir lieu en 1942, ultime illustration de ce style néo-romain devenu fasciste et point final de ce damage que prévoyait Sullivan cinquante ans plus tôt? 


\section{NOTES}

1. « Les grands prix d'architecture en 1900 », La Construction moderne.

2. Huret.

3. Louis H. Sullivan, The Autobiography of an Idea, 1924.

4. The American Architect, février 1899, commentant un article du Moniteur des architectes sur la future Exposition universelle de Paris.

5. Âge doré.

6. Âge d'or.

7. Publiée en 1890 et donc accessible dans les universités américaines à partir de cette date.

8. Charles Moore, Burnham, p. 67.

9. En 1907, un projet de l'architecte Milano pour la salle d'exposition des Beaux-Arts de Rome tend à se rapprocher des dessins de Henry H. Richardson, Louis H. Sullivan et Frank L. Wright.

\section{AUTEUR}

\section{PASCAL LAURENT}

Architecte DPLG, 1989, école d'architecture de Versailles.

EHESS, sous la direction de Bernard Lepetit :

DEA « Territoires urbains », 1994.

Doctorant. 